\title{
Central Limit Theorems for the One-Dimensional Rayleigh Gas with Semipermeable Barriers
}

\author{
L. Erdös ${ }^{1}$ and Dao q. Tuyen ${ }^{2, \star}$ \\ ${ }^{1}$ Eötvös Loránd University, H-1088 Budapest Múzeum krt. 8, Hungary and \\ Princeton University, Department of Mathematics, Fine Hall, Washington Road, Princeton, \\ NJ 08544, USA \\ ${ }^{2}$ Institute of Mathematics of Hanoi, P.O.B. 631, Bo Ho, Hanoi, Vietnam
}

Received August 1, 1990; in revised form July 3, 1991

\begin{abstract}
A version of the one-dimensional Rayleigh gas is considered: a point particle of mass $M$ (molecule), confined to the unit interval [0,1], is surrounded by an infinite ideal gas of point particles of mass 1 (atoms). The molecule interacts with the atoms and with the walls via elastic collision. Central limit theorems are proved for a wide class of additive functionals of this system (e.g. the number of collisions with the walls and the total length of the molecular path).
\end{abstract}

\section{Introduction}

Since the discovery of Brownian motion, it is a great challenge to theoretical physicists and mathematicians to understand its dynamical theory. The chaotic behaviour of small particles in a liquid or in a gas seems to contradict the deterministic laws of the mechanics. Though the mathematical model (Wiener process) was constructed by Wiener and Lévy quite a time ago, the connection between the strict mathematical approach based on microscopic assumptions and the physical reality is far from being completely understood.

The Rayleigh gas, consisting of a heavy Brownian particle (molecule) interacting with an infinite ideal gas of light particles (atoms), seems to be a simple, nonetheless technically quite difficult real mechanical model of the Brownian motion. Here we summarize the known results on this and related models. The common feature of these models is that the randomness of the system is due to the ideal gas, while the possible recollisions give rise to a memory. The qualitative approach of the problem aimed at the ergodic properties of the Rayleigh gas. The simplest one-dimensional case was investigated in [5], where the molecule is confined to a unit interval. This restriction essentially reduced the memory of the system, and rendered possible the proof of the Bernoulli property.

\footnotetext{
* Research partially supported by the Hungarian National Foundation for Scientific Research, grant No. 819/1
} 
The same authors proved later a similar result for a model which contained $N$ molecules ([6]). [In the same paper they considered a two-dimensional model with $N$ hard squares (molecules) as well.] A generalization of their methods to a multidimensional system, where the molecule was a hard ball was obtained by the present authors in [4].

Returning to the one-dimensional case, with the results of [5] at hand, the main task was to remove the walls of the unit interval and let the molecule move on the whole real line. Overcoming several technical difficulties, the authors of [2] could prove the Bernoulliness of the system on the half line. Further generalizations were made in [1], where a constant external force acted on the molecule. Soloveychik has proved the Bernoulliness of the system on the whole real line but in his model the molecule was subject to an external potential $[10,11]$.

A more refined direction of research is aimed at quantitative results, including the estimate of the mean square displacement of the molecule and the convergence to the Wiener process. A good survey of the results and conjectures is found in [15]. Unfortunately the most interesting case (when $M$ is constant) is unsolved (except for the case $M=1$, see $[7,12]$ ), only estimates are known on the mean displacement (see $[9,13])$. In [14] the invariance principle is proved, but only for the case when $M$ grows fast enough with the scaling parameter (remark that the larger $M$ is, the less is the influence of the memory).

In the present paper we prove central limit theorems for the simplest onedimensional model. The memory is reduced in the same way as in [5], i.e. we confine the molecule to the unit interval. We hope this restriction can be eliminated by improvement of the method of Soloveychik et al. (see $[2,10])$. Their key observation is that a.s. there are infinitely many so-called cluster times (by definition: after these times the molecule does not recollide with any atom, with which it collided before). In the model with the walls at 0 and 1 these cluster times naturally arise when the molecule hits the wall 1 . Therefore they can be treated more definitely, while the proof of Soloveychik et al. contains some existence arguments, which makes the direct generalization hard.

As in the majority of particle systems, the memory is so long range that there is no exponential mixing and the Doeblin condition used in a suitable Markov embedding is not uniform. Our main technical tool is a quantitative form of a nonuniform Doeblin condition where we even have aimed at a possibly sharp formation (cf. Sect. 3).

In Sect. 2 we give the formal description of the model and our results. Section 3 contains the basic ideals of our proof and we formulate abstract central limit theorems for a certain class of Markov chains. These theorems are direct generalizations of the classical CLT for Markov chains satisfying the Doeblin condition. In Sects. 4 and 5 we prove that these theorems are applicable for the present model.

\section{The Model and the Results}

Since we are working with the model introduced in [5], we outline its description very briefly. We consider a one-dimensional dynamical system consisting of an infinite number of point particles moving on the nonnegative half-line. A particle of mass $M>1$ (molecule) moves on the unit interval $[0,1]$ and undergoes elastic reflections with the walls at 0 and 1 . The molecule is in contact (via elastic collision) 
with an ideal gas of point particles of mass 1 (atoms) situated to the right of the molecule. The wall in 1 does not affect the atoms.

We suppose that the whole system is in equilibrium at temperature $T=(k \beta)^{-1}$ and density $\varrho=1$. Let $\mathscr{A}=\left(\Omega, \mu, \phi_{t}\right)$ denote our dynamical system, where $\Omega$ is the phase space, $\mu$ is the appropriate Gibbs state and $\phi_{t}$ is the time evolution. A point $\omega \in \Omega$ can be identified with the position and velocity of the molecule $Y_{\omega}=\left(X_{\omega}, V_{\omega}\right)$ $C[0,1] \times \mathbf{R}$ and with the sequence $\left\{x_{\omega}^{n}, v_{\omega}^{n}\right\}_{n=1}^{\infty}$, in which the atoms are labelled in order of increasing position and, for equal position, of increasing velocity $\left(x^{n}\right.$ and $v^{n}$ denote the position and the velocity of the $n^{\text {th }}$ atom).

Clearly the dynamics $\phi_{t}$ generates a stochastic process on $\Omega$, where $\phi_{t} \omega$ will be identified with $Y_{\omega}(t)$ and $\left\{x_{\omega}^{n}(t), v_{\omega}^{n}(t)\right\}_{n=1}^{\infty}$. For brevity, $\omega$ will be omitted if there is no danger of confusion. (We should remark that $\phi_{t}$ is not well defined for all $\omega \in \Omega$ due to the simultaneous collisions, but the set of the exceptional points has a measure zero. For references see the footnote 1 in [5].)

Now we are ready to define our Markov chain, which plays a key role in the present paper. (In choosing the proper chain we were inspired by the approach outlined in the Introduction of [5].) Let $\Sigma$ denote the set of all possible paths (history) of our system in a unit time-interval $I$. Since the dynamics is deterministic, these paths are uniquely determined by the positions and velocities of particles situated in $[0,1]$ at the beginning of $I$, and by those of atoms entered $[0,1]$ during I. Therefore we can identify $\Sigma$ with the following set:

$$
\begin{aligned}
& \sum \sim\left\{Y=(X, V), y=\left(x^{i}, v^{i}\right)_{i=1}^{n}, \bar{y}=\left(x^{n+j}, v^{n+j}\right)_{j=1}^{m}:\right. \\
& \left.n, m \in \mathbf{N}, x^{i} \leqq 1, x^{n+j}>1, x^{n+j}+v^{n+j}<1\right\} \\
& \subset \bigcup_{n=1}^{\infty} \bigcup_{m=1}^{\infty}\left(([0,1] \times \mathbf{R}) \times([0,1] \times \mathbf{R})^{n} \times\left([1, \infty] \times \mathbf{R}_{-}\right)^{m}\right) .
\end{aligned}
$$

Here $Y$ and $y$ corresponds to the molecule and atoms respectively at the beginning of $I$, while $\bar{y}$ describes the atoms entering [0,1] during $I$ (conditions $x^{n+j}>1, x^{n+j}+v^{n+j}<1$ ensure that the corresponding atom enter $[0,1]$ in $I$ ). We can order the particles lexicographically. Again we have to exclude those points from $\Sigma$ which correspond to any irregularity of the system (simultaneous collisions or infinite number of collisions in finite time).

Since $\Sigma$ is a factor space of $\Omega$, it inherits a natural $\sigma$ algebra and a measure $\bar{\mu}$ from $(\Omega, \mu)$. ( $\Sigma$ can be considered as a set of equivalence classes.) Let $S \in \Sigma$, then $\phi_{t} S$ is well defined for $t \in[0,1]$, since the evolution of the system in the time interval $I$ depends only on the information given by $S$.

For all $\omega \in \Omega$ we can define a sequence of random variables $S_{0}(\omega), S_{1}(\omega)$, $S_{2}(\omega) \ldots \in \Sigma$, where $S_{i}(\omega)$ describes the system in $[0,1]$ in the time interval $[i, i+1]$; more formally $S_{i}(\omega)$ is the equivalence class of those points of $\Omega$ which evolve in $[0,1]$ in the same way from time $i$ to time $i+1$, as $\omega$ evolves. Clearly $S_{0}, S_{1}, S_{2}, \ldots$ is a Markov chain with $\Sigma$ as its state space and $\bar{\mu}$ its stationary measure. The Markovity follows from the property of the Gibbs state, i.e. the distribution of the particles entering $[0,1]$ in the time interval $[i, i+1]$ does not depend on the particles entered $[0,1]$ before.

Let $f$ be an arbitrary non-constant measurable function on $\Sigma$ satisfying

$$
\int_{\Sigma}|f(s)|^{4+\delta} d \bar{\mu}(s)<\infty
$$

for a fixed positive $\delta$. 
Now we can formulate our main theorem:

Theorem 1. If $f$ satisfies (1), then the distribution of

$$
\frac{\sum_{i=1}^{n} f\left(S_{i}\right)-n m}{\sigma \sqrt{n}}
$$

approaches the standard normal one, with suitable constants $m$ and $\sigma>0$.

As an easy consequence of our Theorem 1 we have central limit theorems for several interesting additive functionals of our chain. Here we mention four of them. Let $N(T), N_{0}(T), N_{1}(T)$ denote the number of collisions of the molecule with atoms and with the wall 0 and 1 , respectively, until $T$. Furthermore, let

$$
Q(T)=\int_{0}^{T}|V(t)| d t
$$

denote the total length of the molecular path until T. (In all cases the initial particle configuration in $[0,1]$ is distributed according to the stationary measure.)

Corollary 1. For the quantities $N(T), N_{0}(T), N_{1}(T)$ and $Q(T)$ defined above the central limit theorem holds with usual norming.

In the sequel we consider very briefly the related two-sided infinite model. The only alteration is that in this model the atoms are situated on the whole real line. We do not give a formal description, since the state space, the equilibrium measure and the dynamics are defined in a natural way. We can also take the corresponding Markov chain $\left\{S_{i}^{\prime}\right\}_{i=0}^{\infty}$ on an appropriate state space $\Sigma^{\prime}$. Similar to the semi-infinite case we have the following theorem and corollary.

Theorem 2. If $f^{\prime}: \Sigma^{\prime} \rightarrow \mathbf{R}$ is a non-constant measurable function with finite $(4+\delta)^{\text {th }}$ moment with respect to the equilibrium measure, then the distribution of

$$
\frac{\sum_{i=1}^{n} f^{\prime}\left(S_{i}^{\prime}\right)-n m^{\prime}}{\sigma^{\prime} \sqrt{n}}
$$

approaches the standard normal one with suitable $m^{\prime}$ and $\sigma^{\prime}>0$.

Corollary 2. For the appropriate quantities $N^{\prime}(T), N_{0}^{\prime}(T), N_{1}^{\prime}(T)$ and $Q^{\prime}(T)$ defined as in Corollary 1 the central limit theorem holds.

\section{Generalization of Doeblin Condition}

In the standard theory of Markov chains the Doeblin condition guarantees the exponential decay of correlations and the CLT. The Doeblin condition requires that the chain starting from any initial point get out from any "small" set (with respect to a finite measure) after a fixed time with a probability greater than a fixed positive $\varepsilon$. In a formal setting a Markov chain $\left\{S_{i}\right\}_{0}^{\infty}$ satisfies the Doeblin condition if there exists a positive $\varepsilon$, an integer $n$, and a nonzero finite measure $\varphi$ on the state space of the chain such that

$$
\operatorname{Prob}\left(S_{n} \notin B \mid S_{0}=x\right)>\varepsilon
$$


holds for every subset $B$ of the state space with $\varphi(B)<\varepsilon$ (see [3, p. 192]). In our case two phenomena warn us that this condition is not expected to hold. First, if the initial molecular velocity is extremely large, then it cannot be essentially slowed down in a fixed time with a probability greater than $\varepsilon>0$. Second, if the initial configuration contains extremely many slow atoms, then they cannot be "swept out" quickly from the unit interval.

Fortunately, a theorem due to Orey (Theorem 5.1 in [8]) is applicable in our case. For the proof and the background we refer to the original paper; here we summarize only the key notions directly used in our proof.

Orey's paper deals with Markov chains $\left\{S_{i}\right\}_{0}^{\infty}$ on a measurable space $\Sigma$ satisfying the following conditions:

(i) The Borel field of the state space is separable.

(ii) There exists a $\sigma$-finite measure $m$ on the state space such that

Prob(entering $E$ at some time $\left.\mid S_{0}=x\right)=1$,

for all $x \in \Sigma$ and all measurable set $E$ with $m(E)>0$.

Our Markov chain clearly satisfies these conditions with $m=\bar{\mu}$, using the ergodicity of the dynamical system $\mathscr{A}$ (see [5]).

Let $A \subset \Sigma$ be a set of positive $\bar{\mu}$-measure. This set generates the "process on $A$," $S_{A 0}, S_{A 1}, \ldots$, sorting out the elements belonging to $A$ from the initial chain. (Clearly we need the recurrence condition mentioned above for this notion to be well defined.) The conditional measure $\mu_{A}=\bar{\mu}(. \mid A)$ is a stationary measure for the new chain.

The essential point is that choosing the set $A$ in an appropriate way, the process on $A$ satisfies the Doeblin condition.

Let $\tau_{i}$ be the $i^{\text {th }}$ nonnegative integer $\tau$ such that $S_{\tau} \in A$ and let $R_{i}$ be the vector $\left(S_{\tau_{i}+1}, S_{\tau_{i}+2}, \ldots, S_{\tau_{i+1}}\right)(i=1,2, \ldots) . R_{i}$ is a Markov process on the natural state space $\bigcup_{m=1}^{\infty} \Sigma^{m}$. Any function $f$ on $\Sigma$ can be extended to this state space:

$$
f^{*}\left(\left(S_{1}, S_{2}, \ldots, S_{m}\right)\right)=f\left(S_{1}\right)+f\left(S_{2}\right)+\ldots+f\left(S_{m}\right) .
$$

With these notations Theorem 5.1 of [8] asserts that if the process on $A$ satisfies the Doeblin condition, $f$ is nonconstant and $E_{A}\left|f^{*}\left(R_{1}\right)\right|^{2+\delta}<\infty$ (for a fixed $\delta>0$ ) then the distribution of

$$
\frac{\sum_{i=1}^{n} f\left(S_{i}\right)-E_{A}\left(f^{*}\left(R_{1}\right)\right) \bar{\mu}(A) \cdot n}{\sqrt{n \cdot \bar{\mu}(A) \cdot D_{A}^{2}\left(f^{*}\left(R_{1}\right)\right)}}
$$

approaches the standard normal one. (Here $A_{A}$ and $D_{A}^{2}$ denote the expectation and variance with respect to $\mu_{A}$.)

Now we give two abstract generalizations of the Doeblin condition, which are easy consequences of Orey's theorem, but this setting makes clear the connection between them and enables us to use this machinery in the original problem. At the same time these ideas might be useful in similar problems, where the Doeblin condition is satisfied except for a small set. That is why we first formulate a weaker generalization, which is not strong enough in our problem, but helps us to understand the underlying idea.

Given a Markov chain $S_{k}$ on $\Gamma$ with transition probability $P$ and stationary measure $\mu$, which is recurrent in the sense that for all $x \in \Gamma$ and $E \subset \Gamma$, with $\mu(E)>0$, 
there exists an integer $n$ such that $P\left(S_{n} \in E \mid S_{0}=x\right)=1$. (This condition holds if the chain is ergodic.)

Let a function $f$ on $\Gamma$ be given, which has a finite $\left(4+\delta^{\prime}\right)^{\text {th }}$ moment, i.e. $\int_{\Gamma}|f|^{4+\delta^{\prime}} d \mu<\infty$ for a fixed $\delta^{\prime}>0$. Suppose that there is a decreasing sequence of sets $\Gamma_{k}$ in $\Gamma$ such that

$$
\Gamma \supset \Gamma_{1} \supset \Gamma_{2} \supset \ldots, \mu\left(\Gamma_{k}\right) \rightarrow 0,
$$

and the measure of $A:=\Gamma \backslash \Gamma_{1}$ is positive

Proposition 3.1. With the notations and assumptions above, the central limit theorem holds for the sums of type $\sum_{i=1}^{n} f\left(S_{i}\right)$ with suitable norming if there exists an $\varepsilon>0$ such that

(i) $\mu\left(\Gamma_{k}\right) \leqq \frac{\text { const }}{k^{6+\varepsilon}}$,

(ii) $P\left(S_{1} \in A \mid S_{0}\right) \geqq \frac{(5+\varepsilon) \ln k}{k}$ for a.e. $S_{0} \notin \Gamma_{k}$,

(iii) the process on $A$ satisfies the Doeblin condition.

For the application of this theorem in a concrete problem we have to define a "critical order" of each point $x \in \Gamma$ measuring to what extent this point spoils the uniformity in the inequality (2). The set $\Gamma_{k}$ consists of points with critical order of at least $k$. The appropriate definition of $\Gamma_{k}$ should come from analyzing the problem and understanding which phenomena cause the failure of the original Doeblin condition. Then we have to prove that

(i) the set of critical points of order at least $k$ is small;

(ii) from any point of order smaller than $k$ we have a relatively large chance to get into the non-critical set $\left(A=\Gamma \backslash \Gamma_{1}\right)$ in the next step;

(iii) the set $A$ is non-critical indeed, i.e., the process on $A$ satisfies the Doeblin condition.

This theorem can handle those problems, where there is essentially one type of critical behaviour (and a corresponding sequence of critical sets) which prevents us from using the original Doeblin condition. But as we mentioned before, in our problem there are two different phenomena (large molecular velocity and many atoms) which hinder the application of the Doeblin condition. Therefore we need another theorem which involves two different sequences of critical sets. Suppose that there are two decreasing sequence of sets, $\Gamma_{k}$ and $\Lambda_{k}$ in $\Gamma$, such that $\mu\left(\Gamma_{k}\right) \rightarrow 0$, $\mu\left(\Lambda_{k}\right) \rightarrow 0$ and the measure of $A:=\Gamma \backslash\left(\Gamma_{1} \cup \Lambda_{1}\right)$ is positive. Then we have the following proposition.

Proposition 3.2. The statement of Proposition 3.1 holds if there exist numbers $\varepsilon_{k}, \delta_{k}$, $\alpha_{k}$ and $\varrho_{k}$ for each $k$ and an $\varepsilon>0$ such that for $k$ large enough

(i) $\mu\left(\Gamma_{k}\right)<\frac{\text { const }}{k^{6+\varepsilon}}$;

(ii) $\mu\left(\Lambda_{k}\right)<\frac{\text { const }}{k^{6+\varepsilon}}$;

(iii) $\mu\left(S_{1} \in A \mid S_{0}\right) \geqq \varepsilon_{k}$ for a.e. $S_{0} \notin \Lambda_{1} \cup \Gamma_{k}$;

(iv) $\mu\left(S_{\alpha_{k}} \notin \Lambda_{1} \mid S_{0}\right) \geqq \delta_{k}$ for a.e. $S_{0} \notin \Gamma_{k} \cup \Lambda_{k}$;

(v) $\left(1-\varepsilon_{k}\right)^{\varrho_{k}} \leqq \frac{\text { const }}{k^{5+\varepsilon}},\left(1-\delta_{k}\right)^{\frac{k}{\alpha_{k}}-\varrho_{k}} \leqq \frac{\text { const }}{k^{5+\varepsilon}}$;

(vi) the process on A satisfies the Doeblin condition. 
(Suppose that $\varepsilon_{k}, \delta_{k}$ tend to 0 and $\alpha_{k}, \varrho_{k}, \frac{k}{\alpha_{k}}$ tend to infinity as $k \rightarrow \infty$, and $\alpha_{k}$ is integer.)

Remark that Orey's theorem guarantees that the limiting variance is automatically positive, which shows the strength of this method since in many statistical physical problems the proof of the positivity of the diffusion constant requires further efforts.

Proof of Proposition 3.1. In order to apply Orey's theorem we have to prove that conditions (i) and (ii) imply $E_{A}\left|\sum_{i=1}^{\tau} f\left(S_{i}\right)\right|^{2+\delta}<\infty$ for an appropriate $\delta>0$. Using the Cauchy inequality several times we have

$$
\begin{aligned}
E_{A}\left|\sum_{i=1}^{\tau} f\left(S_{i}\right)\right|^{2+\delta} & \leqq E_{A}\left(\tau^{1+\delta} \cdot \sum_{i=1}^{\tau}\left|f\left(S_{i}\right)\right|^{2+\delta}\right) \\
& =\sum_{k=i}^{\infty} E_{A}\left(\left(k^{1+\delta} \cdot \mathbf{1}_{\tau=k}\right)\left(\sum_{i=1}^{k}\left|f\left(S_{i}\right)\right|^{2+\delta}\right)\right) \\
& \leqq \sum_{k=1}^{\infty}\left(E_{A}\left(k^{2+2 \delta} \cdot \mathbf{1}_{\tau=k}\right)\right)^{1 / 2} \cdot\left(E_{A}\left(\sum_{i=1}^{k}\left|f\left(S_{i}\right)\right|^{2+\delta}\right)^{2}\right)^{1 / 2} \\
& \leqq \sum_{k=1}^{\infty} k^{1+\delta} \cdot \mu_{A}(\tau=k)^{1 / 2} \cdot k^{1 / 2} \cdot\left(\sum_{i=1}^{k} E_{A} \mid f\left(\left.S_{i}\right|^{4+2 \delta}\right)^{1 / 2} .\right.
\end{aligned}
$$

Since $\bar{\mu}_{A}(\cdot)=\frac{\bar{\mu}(\cdot \cap A)}{\bar{\mu}(A)} \leqq \frac{\bar{\mu}(\cdot)}{\bar{\mu}(A)}$ we have that

$$
E_{A}\left|f\left(S_{i}\right)\right|^{4+2 \delta} \leqq \frac{1}{\bar{\mu}(A)} \cdot E\left|f\left(S_{i}\right)\right|^{4+2 \delta}=\frac{1}{\bar{\mu}(A)} \cdot E|f|^{4+2 \delta}
$$

by the stationarity. So we get

$$
E_{A}\left|\sum_{i=1}^{\tau} f\left(S_{i}\right)\right|^{2+\delta} \leqq \sqrt{\frac{E|f|^{4+2 \delta}}{\bar{\mu}(A)}} \cdot \sum_{k=1}^{\infty} k^{1.5+\delta} \cdot \mu_{A}(\tau=k)^{1 / 2},
$$

and therefore it is enough to prove that $\mu_{A}(\tau=k+1) \leqq$ const $\cdot \frac{1}{k^{5+3 \delta}}$ for $k \geqq k_{0}$. (Since we need a tail estimate, we deal only with large values of $k$.)

The following inequality is trivial

$$
\begin{aligned}
\mu_{A}(\tau=k+1) \leqq & \mu_{A}\left(\tau=k+1, S_{i} \notin \Gamma_{k} \text { for } \forall i \leqq k\right) \\
& +\mu_{A}\left(\exists i \leqq k: S_{i} \in \Gamma_{k}\right) .
\end{aligned}
$$

The first summand can be estimated by $\left(1-\frac{(5+\varepsilon) \ln k}{k}\right)^{k} \leqq \frac{1}{k^{5+\varepsilon}}$ since $\tau=k+1$ means that we have missed the chance to enter the set $A$ at least $k$ times. The second one is smaller than

$$
\frac{\text { const }}{k^{6+\varepsilon}} \cdot k \cdot \frac{1}{\mu(A)} \leqq \frac{\text { const }}{k^{5+\varepsilon}}
$$

by the stationary. With the choice $\delta=\min \left(\delta^{\prime}, \frac{\varepsilon}{3}\right)$ the Proposition is proved. 
Proof of Proposition 3.2. Following the previous proof, we have to prove that $\mu_{A}(\tau=k+1) \leqq \frac{\text { const }}{k^{5+3 \delta}}$ for a suitable $\delta>0$. It is clear that

$$
\begin{aligned}
\mu_{A}(\tau=k+1) \leqq & \mu_{A}\left(\exists i \leqq k: S_{i} \in \Gamma_{k}\right)+\mu_{A}\left(\exists i \leqq k: S_{i} \in \Lambda_{k}\right) \\
& +\mu_{A}\left(\#\left\{i: i \leqq\left[\frac{k}{\alpha_{k}}\right], S_{i \cdot \alpha_{k}} \notin \Lambda_{1}\right\}<\varrho_{k}\right) \\
& +\mu_{A}\left(\tau=k+1, \#\left\{i: i \leqq\left[\frac{k}{\alpha_{k}}\right], S_{i \cdot \alpha_{k}} \notin \Lambda_{1}\right\} \geqq \varrho_{k}\right) .
\end{aligned}
$$

The first two terms are estimated by $\frac{\text { const }}{k^{5+\varepsilon}}$ as before. The third term is smaller than $\left(1-\delta_{k}\right)^{\frac{k}{\alpha_{k}}-\varrho_{k}}$, since we have missed that chance to enter $\Lambda_{0}$ at least $\left[\frac{k}{\alpha_{k}}\right]-\varrho_{k}$ times of the possible times $\alpha_{k}, 2 \alpha_{k}, 3 \alpha_{k}, \ldots\left[\frac{k}{\alpha_{k}}\right] \cdot \alpha_{k}$. The fourth term is smaller than $\left(1-\varepsilon_{k}\right)^{\varrho_{k}}$, since $\tau=k+1$ means that being in $\Gamma \backslash\left(\Gamma_{k} \cup \Lambda_{1}\right)$ at least $\varrho_{k}$ different times, we have missed the chance to enter $\Gamma \backslash\left(\Gamma_{1} \cup \Lambda_{1}\right)$. From these the proposition follows.

We remark that here we can multiply the estimates of the appropriate probabilities (although the corresponding events are not independent) because (iii) and (iv) give uniform estimates for a.e. $S_{0}$ belonging to the corresponding set.

We also remark that similar propositions can be proved for the case of more than two sequences of critical sets.

\section{Proof of Theorem 1}

Now we have to define the suitable sequences of sets $\Gamma_{k}$ and $\Lambda_{k}$. They correspond to the large molecular velocity and the large number of atoms in $[0,1]$, respectively. Let

and

$$
\Gamma_{k}=\left\{S \in \Sigma:|V(S)|>\psi_{k}\right\}
$$

$$
\Lambda_{k}=\left\{S \in \Sigma: n(S)+m(S)>\varphi_{k}\right\},
$$

where $V(S), n(S)$, and $m(S)$ denote the molecular velocity, the number of atoms in $[0,1]$ at the beginning of the unit time interval and the number of entering atoms, respectively (remember that $S$ corresponds to a specific history of the system restricted to $[0,1]$ during a unit time interval, see Sect. 2). The number $\psi_{k}$ and $\varphi_{k}$ will be determined later $\left(\psi_{k} \rightarrow \infty, \varphi_{k} \rightarrow \infty\right.$ and $\varphi_{k} \in \mathbf{N}$ are assumed), but remark that their values for some small indices $k$ do not influence the conditions (i)-(iv), since they are required to fulfill only for $k$ large enough. In the following proofs it will not be emphasized but always assumed that all estimates are tail estimates, i.e., they are valid only for $k$ large enough. Therefore it can be assumed that $A=\Gamma \backslash\left(\Gamma_{1} \cup \Lambda_{1}\right)$ consists of those points $S \in \Sigma$ for which $n(S)=m(S)=0$ (there is no atom in $[0,1]$ in the time interval $I=[0,1])$, and $|V(S)| \leqq K(K$ is a large constant to be determined later). We remark that $n(S)=m(S)=0$ guarantees that $|V|$ is constant in $I$. Clearly $\bar{\mu}(A)>0$. First we verify the condition (vi), since its proof makes transparent how we can control the development of the system. 


\section{Lemma 4.1 The process on A satisfies the Doeblin condition.}

Proof. Let $\bar{\mu}$ be the finite measure in the definition of Doeblin condition. Let $S_{0} \in A$ be an arbitrary point and denote $Y(1)=(X(1), V(1))$ the position and velocity of the molecule at time $1(|V(1)| \leqq K$, since the absolute value of the molecular velocity remains unchanged if there is no atom). We prove that there exists a positive $\varepsilon=\varepsilon(K)$ (depending only on $K$ ), such that for every $B \subset A, \bar{\mu}(B) \leqq \varepsilon(K) S_{1} \notin A$ and $S_{2} \in A \backslash B$ with probability at least $\varepsilon(K)$. This would mean that the process on $A$ satisfies the Doeblin condition with $\varepsilon=\varepsilon(K), n=1$ and $\varphi=\bar{\mu}$, since $S_{A 0}=S_{0}$, $S_{A 1}=S_{2}$ and $\operatorname{Prob}\left(S_{A 1} \notin B \mid S_{A 0}\right)>\varepsilon(K)$. (By the strong Markov property we can replace the random indices by deterministic ones on a suitable subset.)

Let an atom $\alpha$ enter $[0,1]$ at time $t, 1 \leqq t \leqq 2$, with velocity $v<0$, and no other atom enter $[0,1]$ in the time interval $[1,3]$. Clearly $S_{1} \notin A$, and for $S_{2} \in A$ it is enough to guarantee that $\alpha$ leave $[0,1]$ before time 2 , and that $|V(2)| \leqq K$.

For a given $Y(1)$ we have a map

$$
\Phi_{Y(1)}:[1,2] \times \mathbf{R}_{-} \mapsto[0,1] \times \mathbf{R},
$$

where

$$
\Phi_{Y(1)}(t, v)=\left(X_{Y(1) \cup \alpha}(2), V_{Y(1) \cup \alpha}(2)\right)
$$

denotes the molecular position and velocity at time 2 if $\alpha$ enters $[0,1]$ only. Notation $Y(1) \cup \alpha$ indicates that only $Y(1)$ and $\alpha$ have influence on the molecular position and velocity at time 2 . We would like to ensure "sufficient spreading" of $Y(2)$ for any given $Y(1)$. The randomness is in $\alpha$ and its parameters $(t, v)$ have positive (separated from zero) density. Therefore if $\Phi_{Y(1)}$ is not close to a singular transformation then we get a positive (moreover separated from zero) density for $Y(2)$ on a large set. Thus the distribution of $Y(2)$ cannot be concentrated on a small set. Since $S_{2}$ is uniquely determined by $Y(2)$, this will give the result.

We distinguish two cases.

First case. $|V(1)|<5$. In this case one can find a time $t_{1}$ such that $1.3 \leqq t_{1} \leqq 1.5$, and the molecule would more freely (without any collision) between times $t_{1}$ and $t_{1}+0.1$ if there were no atom $\alpha$. (The bounds 1.3 and 1.5 guarantee that $\alpha$ should have entered $[0,1]$ after time 1 , and it leaves this interval before time 2.) Elementary calculations show that if $K$ is large enough then there exist two positive constants, $c_{1}$ and $c_{2}$, depending on $K$, such that if $c_{1}<-v<c_{2}$ and $\alpha$ hits the molecule between $t_{1}$ and $t_{1}+0.1$, then the post-collision velocity of the atom is so large that it leaves $[0,1]$ before time 2 , it does not recollide with the molecule (since $c_{1}<-v$ and $|V(1)|<5$ ), and the post-collision molecular velocity is smaller than $K$ in absolute value (due to $-v<c_{2}$ ). Moreover, $c_{1}$ can be chosen so large that $\alpha$ should enter $[0,1]$ after time 1 .

The condition that $\alpha$ hits the molecule between $t_{1}$ and $t_{1}+0.1$ gives an interval of length 0.1 for the choice of $t$ for any $v$ satisfying $c_{1}<-v<c_{2}$. The set of all $(t, v)$ satisfying these conditions is obviously a subset $H_{0}=H_{Y(1)} \subset[1,2] \times \mathbf{R}_{-}$of positive $v$ measure $\left(v\right.$ is the conditional measure on $[1,2] \times \mathbf{R}_{-}$inherited from the distribution of the ideal gas under the condition that only $\alpha$ entered $[0,1]$ in the time interval $[1,3])$. Remark that $v\left(H_{0}\right)$ does not depend on $Y(1)$.

It follows from the construction above that $\Phi_{Y(1)}$ is piecewise differentiable on $H$ since the curves $\Phi_{Y(1)}^{-1}(0, \cdot)$ and $\Phi_{Y(1)}^{-1}(1, \cdot)$ divide $H_{0}$ into finitely many pieces on which $\Phi_{Y(1)}$ is differentiable. Let $H$ be one of these pieces of positive measure. 
Moreover, by elementary calculation, its Jacobian is uniformly separated from zero: $\left|J a c \Phi_{Y(1)}\right| \geqq c_{K}>0$. The bound $c_{K}$ does not depend on $Y(1)$ and $c_{K}<1$ can be assumed.

The points of $[0,1] \times \mathbf{R}$ can be identified by those points of $\Sigma$ which contain no atoms $(k=m=0)$. Therefore $\Phi_{Y(1)}(H)$ can be identified with a subset of $\Sigma$ [moreover $\left.\Phi_{Y(1)}(H) \subset A\right]$, and the condition on the Jacobian guarantees that $\bar{\mu}_{A}\left(\Phi_{Y(1)}(H)\right)>0$ uniformly. Moreover, $\bar{\mu}_{A, \alpha}\left(\Phi_{Y(1)}(H)\right)$ is also uniformly positive, where $\bar{\mu}_{A, \alpha}$ denotes the conditional measure derived from $\bar{\mu}_{A}$ under the condition that only one atom $(\alpha)$ entered $[0,1]$ between times 1 and 3 . By the way, for any $\bar{H} \subset H$ we have that $v(\bar{H})=\bar{\mu}_{A, \alpha}\left(\Phi_{Y(1)}(\bar{H})\right)$, since $\Phi_{Y(1)}$ is bijective and both measures derive from the same Gibbs state.

Now if we choose $\varepsilon(K)<\frac{1}{2} c_{K} \cdot \min \left(\bar{\mu}_{A, \alpha}\left(\Phi_{Y(1)}(H)\right), v(H)\right)$, then for any subset $A_{0} \subset A, \bar{\mu}_{A}\left(A_{0}\right)<\varepsilon(K)$, the $v$ measure of its pre-image $\Phi_{Y(1)}^{-1}\left(A_{0}\right)$ will be smaller than

$$
\frac{1}{c_{K}} \cdot \varepsilon(K)<\frac{v(H)}{2} .
$$

Therefore starting from any $Y(1)$ we get out of any fixed set $A_{0}$ of measure smaller than $\varepsilon(K)$ with probability greater than

$$
\frac{v(H)}{2} \geqq \frac{1}{c_{K}} \cdot \varepsilon(K)>\varepsilon(K) .
$$

Second case. $|V(1)| \geqq 5$. In this case there exists a time $t_{2}$ such that $1.1 \leqq t_{2} \leqq 1.7$ and $X\left(t_{2}\right)=0$ if no atom entered. (The bounds 1.1 and 1.7 stand for the same reason as in the first case.) This guarantees that the molecule would move freely between times $t_{2}$ and $t_{2}+\frac{1}{K}$, and this time interval should be taken for the collision with $\alpha$.

Again elementary calculations show that the parameters $(t, v)$ of $\alpha$ can be chosen in such a way that

$-\alpha$ should not enter $[0,1]$ before time 1

- $\alpha$ leaves $[0,1]$ before time 2

- the post-collision molecular velocity is smaller than $K$ in absolute value.

The first requirement involves that $-v \geqq 10$, the second one is automatic and the third one involves an upper bound for $-v$ depending on $K$.

Similarly to the first case, there exists a set $H \subset[1,2] \times \mathbf{R}_{-}$of positive $v$ measure such that if $(t, v) \in H$ then these conditions are satisfied. From this point the proof is analogous to that of the first case.

Now we go on verifying the conditions of Proposition 3.2. Numbers $\psi_{k}$ will be determined in such a way that (i) be satisfied. For this purpose we need the following lemma.

Lemma 4.2. If at time 1 only the molecule were in $[0,1]$ and $|V(1)| \leqq K$, and if for any $i|V(i)| \geqq \psi_{k}>K$, then there was an atom of velocity greater than $\psi_{k}$ (in absolute value) entering $[0,1]$ between times 1 and $i$.

This lemma points out an essential property of the physical system. Namely, it asserts that it is impossible to speed up the molecule by many, but relatively show atoms.

Consider the collision when the molecule first gains its greatest velocity $|V|_{\max }$ (in absolute value) between 1 and $i$. It should be a collision with an atom. Denote 
with $v_{-}, v_{+}, V_{-}$, and $V_{+}$the pre- and postcollision velocities of the atom and the molecule, respectively $\left(\left|V_{+}\right|=|V|_{\max }\right)$. The collision equations are the following:

$$
\begin{gathered}
v_{+}=-\kappa v_{-}+(1+\kappa) V_{-}, \\
V_{+}=\kappa V_{-}+(1-\kappa) v_{-},
\end{gathered}
$$

(where $\left.\kappa=\frac{M-1}{M+1}\right)$.

From these it follows that $\left|V_{+}\right| \leqq \max \left(\left|V_{-}\right|,\left|v_{-}\right|\right)$. Clearly $\left|V_{-}\right|<\left|V_{+}\right|$, therefore $\left|v_{-}\right| \geqq\left|V_{+}\right| \geqq|V(i)| \geqq \psi_{k}$. Since before the collision the molecular velocity was smaller than $\left|V_{+}\right| \leqq\left|v_{-}\right|$, the molecule never collided with this atom before (between any two recollisions the molecular path is longer than the path of the atom). Therefore this atom arrived from outside with a negative velocity $v_{-}$greater than $\psi_{k}$ in absolute value.

Explicit computation shows that the probability of the occurrence of an atom with velocity greater than $\psi_{k}$ (in absolute value) between 1 and $k+1$ is smaller than

$$
k \cdot \sqrt{\frac{\beta}{2 \pi}} \cdot \int_{\psi_{k}}^{\infty} e^{-\frac{\beta v^{2}}{2}} d v \leqq \text { const } \cdot k \cdot e^{-\frac{\beta \psi_{k}^{2}}{2}} .
$$

This quantity is smaller than const $\cdot \frac{1}{k^{6+\varepsilon}}$ if $\psi_{k} \geqq \sqrt{\frac{14+2 \varepsilon}{\beta}} \cdot \sqrt{\ln k}$. Let us fix $\varepsilon=1$ and then $\psi_{k}=\sqrt{\frac{16}{\beta}} \cdot \sqrt{\ln k}$.

For the definition of $\varphi_{k}$ we use condition (ii). For large $k$, the event $\Lambda_{k}$ is highly unlikely since $\bar{\mu}$ is inherited from the stationary Gibbs state which strongly suppresses the particle condensation (due to the fast decaying tail of the Poisson distribution). Clearly both $n(S)$ and $m(S)$ have Poisson distribution, therefore

$$
\begin{aligned}
\bar{\mu}\left(\Lambda_{k}\right) & =\bar{\mu}\left(S \in \Sigma: n(S)+m(s)>\varphi_{k}\right) \\
& \leqq \bar{\mu}\left(n(S)>\frac{\varphi_{k}}{2} \text { or } m(S)>\frac{\varphi_{k}}{2}\right) \leqq \frac{\text { const }}{\left(\frac{\varphi_{k}}{2}\right) !},
\end{aligned}
$$

which is smaller than $\frac{\text { const }}{k^{7}}$ if $\varphi_{k} \geqq$ const $\cdot \ln k$ for any positive constant. Let us choose $\varphi_{k}=\left[c_{0} \cdot \ln k\right]$, where the constant $c_{0}>0$ will be specified later.

For the condition (iii) we give a good lower estimate for the following probability:

$$
\bar{\mu}\left(S_{1} \in A \mid S_{0}\right) \geqq \varepsilon_{k}
$$

if $K<\left|V\left(S_{0}\right)\right|<\psi_{k}$ and $n\left(S_{0}\right)=0$. ( $\varepsilon_{k}$ will be of order $e^{-\ln \ln k \cdot \ln \ln \ln k}$.)

Let $t_{0}$ denote the first molecular collision time with the wall 0 after time 0 , and let $t_{1}$ be the first collision time with the wall 1 after $t_{0}$ if there were no entering atoms. Since $\left|V\left(S_{0}\right)\right|>K>10$ we have that $t_{1}<i+0.3$. Clearly $t_{1}-t_{0}=\frac{1}{\left|V\left(S_{0}\right)\right|}$ $>\frac{1}{\psi_{k}}$. Let $\bar{t}_{1}=t_{0}+\frac{1}{\psi_{k}}$, then $\bar{t}_{1} \leqq t_{1}$. Between times $t_{0}$ and $\bar{t}_{1}$ we direct $n$ atoms to the unit interval with negative velocities $v_{1}, v_{2}, \ldots, v_{n} \geqq-1$ (the labelling corresponds to the order of collisions). Let $V_{1}=\left|V\left(S_{0}\right)\right|, V_{2}, V_{3}, \ldots, V_{n}$ denote the molecular velocities before the collisions, and let $V_{n+1}$ denote the final molecular velocity $\left(\left|V_{n+1}\right|=\left|V\left(S_{1}\right)\right|\right)$. 
The number of atoms and their velocities will be chosen in such a way that $V_{n+1}$ is the first molecular velocity smaller than $K$ in absolute value, $V_{1}, V_{2}, \ldots, V_{n}$ is positive and the atoms leave $[0,1]$ before time $i+1$ without any recollision.

Iterating the collision equation $V_{i+1}=\kappa V_{i}+(1-\kappa) v_{i}$ we have

$$
V_{n+1}=\kappa^{n} V_{1}+(1-\kappa)\left(\kappa^{n-1} v_{1}+\kappa^{n-2} v_{2}+\ldots+v_{n}\right) .
$$

If we choose $n$ so large that $\kappa^{n} V_{1}<K$, i.e. $n=\left[\frac{\ln K-\ln V_{1}}{\ln \kappa}\right]+1$, then clearly $V_{n+1}<K$, and

$$
V_{n+1} \geqq \kappa^{n} V_{1}-(1-\kappa)\left(\kappa^{n-1}+\ldots+1\right) \geqq-K
$$

would hold. Therefore $\left[\frac{\ln K-\ln V_{1}}{\ln \kappa}\right]+1$ is an upper estimate for $n$.

Moreover, if $K$ is large enough $(K>1)$, then the post-collision velocities of the atoms $\left(v_{i}^{+}\right)$are greater than $K$ [since $v_{i}^{+}=-\kappa v_{i}+(1+\kappa) V_{i}$ and $\left|V_{i}\right|>K, v_{i}<0$, $\left.\left|v_{i}\right| \leqq 1\right]$, therefore they leave $[0,1]$ before time $i+1$. Easy calculation shows that no recollision occurs between times $i$ and $i+1$. So the probability in (5) can be estimated by the probability of that event that exactly $n$ atoms enter $[0,1]$ in the time interval $\left[t_{0}, \bar{t}_{1}\right]$ of length $\frac{1}{\psi_{k}}$, their velocities are between 0 and -1 , and except for these atoms no other atom enters $[0,1]$ between times 0 and 2 (this needs to guarantee $S_{1} \in A$ ).

Direct computation shows that this probability is $e^{-\lambda} \cdot \frac{\lambda^{n}}{n !} \cdot e^{-(2-\lambda)}$, where

$$
\lambda=\frac{1}{\psi_{k}} \cdot \sqrt{\frac{\beta}{2 \pi}} \cdot \int_{-1}^{0} \mathrm{e}^{-\frac{\beta v^{2}}{2}} d v=\frac{\text { const }}{\sqrt{\ln k}} .
$$

Clearly this probability decreases as $n$ increases, therefore the worst case is when $V_{1}=\psi_{k}$ and $n$ is equal to its upper bound given above (supposing that $\left.\psi_{k}>2 K\right)$. Then the uniform lower estimate for $\bar{\mu}\left(S_{1} \in A \mid S_{0}\right)$ is $\varepsilon_{k}=e^{-2} \cdot \frac{\lambda^{n_{0}}}{n_{0} !}$, where

$$
n_{0}=\left[\frac{\ln \psi_{k}-\ln K}{-\ln \kappa}\right]+1 \leqq \mathrm{const} \cdot \ln \ln k .
$$

Now we choose $\varrho_{k}$ so that the first inequality of (v) be satisfied. Elementary calculations show that $\left(1-\varepsilon_{k}\right)^{\varrho_{k}} \leqq$ const $\cdot \frac{1}{k^{6}}$ if $\varrho_{k} \geqq e^{2(\ln \ln k)^{2}}$. Fix $\varrho_{k}=e^{2(\ln \ln k)^{2}}$. Remark that $\varrho_{k}$ tends to infinity slower than any power of $k$.

Finally we have to specify $\alpha_{k}$ and $\delta_{k}$ for the estimate (iv). Let $\alpha_{k}=2 \varphi_{k}$ and $\delta_{k}=\tilde{c}_{0}^{\alpha_{k}}$, where $\tilde{c}_{0}$ is fixed. Easy calculation shows that if $c_{0}$ is small enough depending on $\tilde{c}_{0}$, then the second inequality in (v) is also satisfied.

In the sequel we concentrate on the estimate (iv). Denote with $\Omega[0,1]$ the state space of all configurations of particles in $[0,1]$ with the natural measure $\tilde{\mu}_{A}$ inherited from the Gibbs state $\mu_{A}$. Let $Z(i) \in \Omega[0,1]$ describe the positions and velocities of particles in $[0,1]$ at time $i$. This is clearly Markovian. Let the function $n: \Omega[0,1] \rightarrow \mathbf{N}$ count the number of atoms in $[0,1]$ that is $n(Z(i))=n\left(S_{i}\right)$ with our previous notation. 
Suppose that all particles in $[0,1]$ have a small velocity. Then a not too fast outside atom pushes the molecule against the wall at 0 , and flies away from $[0,1]$. After the reflection, the molecule obtains a positive velocity. With this velocity it can push out some atoms from $[0,1]$, and, at the same time, it slows down quickly. This can happen during a time interval of length 1 with a constant probability. If this happens in $\frac{\alpha_{k}}{2}$ time intervals of length 1 between 0 and $\alpha_{k}$, then the number of atoms in $[0,1]$ decreases by at least $\varphi_{k}=\frac{\alpha_{k}}{2}$, i.e. this number becomes zero.

Now let us fix a configuration $Z(0)$ in $[0,1]$ at time 0 with $n(Z(0)) \leqq \varphi_{k}$. Let $E$ be the following event:

- For each $i=0,1,2, \ldots, \varphi_{k}+1$ exactly one atom entered $[0,1]$ with a negative velocity smaller than $\min \left(-20(1+\kappa),-\frac{28}{1-\kappa},-\frac{36}{\kappa}\right)$ in the time interval $(\bar{t}+i$, $\bar{t}+i+0.4)$, where $\bar{t}=\varphi_{k}-2$.

- No other atom entered $[0,1]$ between times 0 and $\alpha_{k}$. Clearly

$$
\mu(E)=c_{2}^{\varphi_{k}+2} \cdot c_{3}^{\alpha_{k}-\left(\varphi_{k}+2\right)} \geqq \tilde{c}_{0}^{\alpha_{k}},
$$

where $\tilde{c}_{0}=\min \left(c_{2}, c_{3}\right)$. Constants $c_{2}$ and $c_{3}$ depend only on $\beta$ and $M$. We prove that on the event $E$ we have $n\left(Z\left(\alpha_{k}\right)\right)=0$ if $n(Z(0)) \leqq \varphi_{k}$, i.e. $\delta_{k}=\tilde{c}_{0}^{\alpha_{k}}$ is a suitable estimate for (iv).

We need the following elementary propositions to formalize the idea outlined above. Since the proofs consist of elementary calculations, we omit them.

Proposition 4.1. If there is no atom in $[0,1]$ at some time $t$, then any atom entering $[0,1]$ at time $t$ with absolute velocity greater than $20(1+\kappa)$ leaves $[0,1]$ before $t+0.4$, supposing that no other atom enters until $t+0.3$.

The next proposition describes the situation in $[0,1]$ at time $\bar{t}=\varphi_{k}-2$ on the event $E$. Clearly $Z(\bar{t})$ contains at most $\varphi_{k}$ atoms.

Proposition 4.2. On the event $E$ at least one of the following assertions holds for $Z(\bar{t})$ :

(A) $Z(\bar{t})$ contains no atom;

(B) each particle in $Z(\bar{t})$ has a velocity smaller than 8 in absolute value.

Proposition 4.3. Suppose that at some time $t$ we have $n(Z(t)) \geqq 1,|V(t)| \leqq 8$ and no atoms in $[0,1]$ have a negative velocity greater than 8 in absolute value. Assume that between $t$ and $t+1$ no outside atom entered $[0,1]$ except for $\gamma$, which entered between times $t$ and $t+0.4$ with a negative velocity smaller than $\min \left(-\frac{28}{1-\kappa},-\frac{36}{\kappa}\right)$. Then $n(Z(t+1)) \leqq n(Z(t))-1$, and at time $t+1$ either $|V(t+1)| \leqq 8$ and no atoms in $[0,1]$ have a negative velocity greater than 8 in absolute value, or there remains no atom in $[0,1]$.

Now suppose that $n(Z(0)) \leqq \frac{\alpha_{k}}{2}=\varphi_{k}$, and we prove that $n\left(Z\left(\alpha_{k}\right)\right)=0$ on the event E. By Proposition 4.2 (A) or (B) holds.

If (A) holds, then by Proposition 4.1 each atom entering between $\bar{t}+i$ and $\bar{t}+i+0.4\left(i=0,1, \ldots, \varphi_{k}+1\right)$ leaves $[0,1]$ before $\bar{t}+i+1$. Therefore there is no atom in $[0,1]$ at integer times after $t$, thus $n\left(Z\left(\alpha_{k}\right)\right)=0$. 
If (B) holds then iterating Proposition 4.3 and using Proposition 4.1 we have that

$$
n(Z(\bar{t}+i)) \leqq \max (0, n(Z(\bar{t}))-i)
$$

Since $n(Z(\bar{t})) \leqq n(Z(0)) \leqq \varphi_{k}$, we get

$$
n\left(Z\left(\alpha_{k}\right)\right) \leqq n\left(Z\left(\bar{t}+\varphi_{k}+1\right)\right) \leqq \max \left(0, \varphi_{k}-\left(\varphi_{k}+1\right)\right)=0 .
$$

This accomplishes the proof of Theorem 1.

\section{Moment Estimations}

In this section we prove that the fifth moment of the random variables $N(1), N_{0}(1)$, $N_{1}(1)$ and $Q(1)$ are finite, i.e. we prove Corollary 1 (for notations see Sect. 2).

Let $R$ denote the number of atoms entering $[0,1]$ between times 0 and 1 .

Lemma 5.1. With the notations above the following inequalities hold:

(i) $N_{0}(1) \leqq N_{1}(1)+R+1$,

(ii) $N(1) \leqq N_{0}(1) \cdot R$,

(iii) $N_{1}(1) \leqq 2 Q(1)+2$.

Proof. (i) Let $\gamma$ an atom entering $[0,1]$. Clearly it can collide with the molecule several times. The key observation is that $\gamma$ can change the sign of the molecular velocity only at most one. It is due to the fact that, if $\gamma$ changes the sign of molecular velocity, then it has a negative pre- and a positive post-collision velocity, and clearly the velocity of $\gamma$ cannot decrease. Thus the inequality (i) follows, since between two collisions with the wall 0 there should happen a change in the sign of the molecular velocity, i.e. a collision with the wall 1 or with an atom.

(ii) This inequality is the consequence of the fact that between two collisions with the same atom, the molecule should collide with the wall 0.

(iii) Trivial.

Since the distribution of $R$ is Poisson, its moments are finite. Using Holder and Cauchy inequalities several times and the previous lemma, for the finiteness of the fifth moment of $N(1), N_{0}(1), N_{1}(1)$ and $Q(1)$ it is enough to prove that $E(Q(1))^{10}<\infty$. For this purpose we prove that the distribution of $Q(1)$ has an exponentially decaying tail. If $Q(1) \geqq c$, then at some time between 0 and 1 the molecular velocity has to be greater than $c$ (in absolute value). But using the idea of Lemma 4.2, we have that it is impossible, unless the molecular velocity at time 0 were greater than $c$, or an atom faster than $c$ entered $[0,1]$. But obviously both events have exponentially small probability with respect to the equilibrium distribution.

\section{Outline of the Proof of Theorem 2 and its Corollary}

Since the proofs for the one- and two-sided models are essentially the same, we only point out the nontrivial alterations.

In the proof of Lemma 5.1 we constructed a set of suitable outside environments in the time interval $[1,3]$. Now we should add to this construction the requirement that no atom enter $[0,1]$ in this time interval from the left. The measure of the suitable set becomes smaller but remains positive.

The same trick works in the construction of environments for the estimations of (iii) and (iv); we take a supplementary condition that no atom entered from the left. It modifies the constants by a factor corresponding to the probability that no atom entered from left, but it does not change the estimates. 
The inequality (i) of Lemma 4.1 remains valid if we denote with $R$ the number of all entering atoms. Instead of inequalities (ii) and (iii) the following ones are true:

(ii) $N^{\prime}(1) \leqq\left(N_{1}^{\prime}(1)+N_{0}^{\prime}(1)+R\right) \cdot R$,

(iii) $N_{0}^{\prime}(1)+N_{1}^{\prime}(1)<2 Q^{\prime}(1)+2$.

These inequalities also imply the necessary moment estimates.

\section{Some Remarks on the Multidimensional Case}

Consider the multidimensional equivalent of the present system, which is described and investigated in [4]. In this model we confine the molecule, which is a hard ball (with positive radius), to a convex compact domain with a smooth boundary. This boundary serves as a semipermeable barrier, i.e. it does not affect the motion of the point particles of the multidimensional ideal gas, but it reflects the molecule. As the investigations on the Bernoulli property of the onedimensional model of [5] has led to the multidimensional generalization, the question naturally arises how one should modify the present proof to obtain a similar central limit theorem for the multidimensional model.

Attentively reading this proof, one can easily define a suitable set $A$ (no atoms in the domain and bounded molecular velocity) and prove that the process on $A$ satisfies the Doeblin condition. For this purpose one can use the idea of Lemma 3 in [4], i.e. to compute that collisions with two suitably entering atoms yield a full rank perturbation in the phase point of the molecule. Since the precollision molecular velocity is bounded, it can be proved that the Jacobian of this perturbation is uniformly bounded away from zero. This yields a sufficient uniform perturbation for the phase point of the molecule, which assures the Doeblin condition (see Lemma 4.1).

Qualitative examinations show that in the multidimensional case not only two but three phenomena appear which hinder the Doeblin condition. Besides the large molecular velocity and the large quantity of atoms in the bounded domain, any configuration containing a slow atom very close to the boundary of the domain also involves a long memory decay. In this case the problem comes from the geometry of the system: it is very unlikely to hit an atom close to the boundary by a relatively large ball, since the ball gets reflected from the wall before it would reach the atom. This reasoning supports the idea that the state space should be divided by three different sequences of sets. At the present time we are able to give effective estimates only for the sets describing the large molecular velocity [similar to (iii)], and we cannot treat effectively the other two phenomena, but we are convinced that this can be done (the higher the dimension, the smaller the chance of recollisions, which should reduce the memory). Until this progress is not made, there is no point in giving more technical details about the previous steps outlined above.

Acknowledgements. The authors are very grateful to D. Szász and A. Krámli for valuable discussions and their encouragement. We are also indebted to D. Dürr for his comments and suggestions.

\section{References}

1. Boldrighini, C.: Bernoulli property for a one-dimensional system with localized interaction. Commun. Math. Phys. 103, 499-514 (1986)

2. Boldrighini, C., Pellegrinotti, A., Presutti, E., Sinai, Ya.G., Soloveychik, M.R.: Ergodic properties of a semi-infinite one-dimensional system of statistical mechanics. Commun. Math. Phys. 101, 363-382 (1985)

3. Doob, J.: Stochastic processes. New York: Wiley 1953 
4. Erdös, L., Tuyen, D.Q.: Ergodic properties of the multidimensional Rayleigh gas with a semipermeable barrier. To appear in J. Stat. Phys.

5. Goldstein, S., Lebowitz, J.L., Ravishankar, K.: Ergodic properties of a system in contact with a heat bath: a one dimensional model. Commun. Math. Phys. 85, 419-427 (1982)

6. Goldstein, S., Lebowitz, J.L., Ravishankar, K.: Approach to equilibrium in models of a system in contact with a heat bath. J. Stat. Phys. 43, 303-315 (1986)

7. Harris, T.E.: Diffusions with collisions between particles. J. Appl. Probab. 2, 323-338 (1965)

8. Orey, S.: Recurrent Markov chains. Pacific J. Math. 9, 3, 805-827 (1959)

9. Sinai, Ya.G., Soloveychik, M.R.: One-dimensional classical massive particle in the ideal gas. Commun. Math. Phys. 104, 423-443 (1986)

10. Soloveychik, M.R.: Ergodic properties of a system of statistical mechanics with an external potential (in Russian). Izv. Akad. Nauk SSSR Ser. Mat. 53, 1, 179-199 (1989)

11. Soloveychik, M.R.: Sufficient conditions for Bernoulliness of a $K$-system and its application in the classical statistical mechanics (in Russian). Mat. Zametki 45, 2, 105-112 (1989)

12. Spitzer, F.: Uniform motion with elastic collisions of an infinite particle system. J. Math. Mech. 18, 973-989 (1969)

13. Szász, D., Tóth, B.: Bounds for the limiting variance of the "heavy particle" in $\mathbf{R}^{1}$. Commun. Math. Phys. 104, 445-455 (1986)

14. Szász, D., Tóth, B.: Towards a unified dynamical theory of the Brownian particle in an ideal gas. Commun. Math. Phys. 111, 41-62 (1987)

15. Szász, D., Tóth, B.: A dynamical theory of Brownian motion for the Rayleigh gas. J. Stat. Phys. 47, 681-693 (1987)

\section{Communicated by J. L. Lebowitz}

Note added in proof. Misha Soloveychik has pointed out that the positivity of the limiting variance $\sigma$ in Theorem 1 is not necessarily true. It turned out that Orey's theorem (Theorem 5.1 in [8]) we were using was incomplete. Orey used the usual CLT for Markov chains satisfying the Doeblin condition, but seemingly he did not check the positivity of the limiting variance of the sum. Therefore an extra condition is needed in his theorem. This mistake has the consequence that in our Theorems 1 and 2 the positivity of $\sigma$ and $\sigma^{\prime}$ does not follow automatically. We expect that it is true for all functions except for some trivial counterexamples where the sum $\sum f\left(S_{i}\right)$ remains bounded (e.g. if $f$ denotes the signed molecular displacement - the counterexample given by Soloveychik).

We can show this positivity for a certain class of functions including the total molecular path $Q(T)$ and the numbers of collisions $N_{0}(T)$ and $N_{1}(T)$. In the sequel we outline briefly the argument and the necessary changes in the setup. We omit all technical and formal details.

First we have to modify the Markov chain. So far an element of the chain described the history of the system in $[0,1]$ in a unit time interval, i.e. we cut the time axis into pieces of length 1 at points $t_{n}=n(n$ is integer). Now we modify this sequence of cuts $t_{n}$ : if the molecular velocity is larger than 4 in absolute value and there is no atom in $[0,1]$ during the time interval $\left[t_{n}, t_{n}+1\right]$, then we put the next cut $t_{n+1}$ at the last point on the time axis when the molecule reached the wall at 1 before $t_{n}+1$.

It can be easily checked that this modification does not essentially effect Sect. 4 . The CLT for this new chain gives asymptotic normality with norming $\sqrt{n}$, although the corresponding norming should be $\sqrt{t_{n}}$ considering the time up to the $n^{\text {th }}$ step. But the ergodicity of the chain guarantees that $t_{n} / n$ converges almost surely to a positive number which proves the asymptotic normality with the norming $\sqrt{t_{n}}$, too.

Finally, for the positivity of the variance one has to prove that

$$
\liminf \frac{1}{n} D^{2}\left(\sum_{i=1}^{n} f\left(S_{i}\right)\right)>0
$$

Let $B$ denote the following subset of the new state space: $B=\{$ there is no atom in $[0,1],|V| \geqq 4$, the molecule starts at the wall at 1$\}$. According to the modification, all paths corresponding to an element in $B$ end at the wall at 1 , and it is also clear that $\mu(B)>0$. The key observation is that if $S_{\imath} \in B$ and no atom enters $[0,1]$ in the next unit of time then $S_{i+1}=S_{i}$. On the other hand, the number $N_{j}$ of consecutive elements of the chain falling into $B$ at the $j^{\text {th }}$ occasion is totally independent of the history of the chain before and the ideal gas, so $N_{j}$ is a Poisson random variable and $D^{2}\left(N_{j}\right)>0$.

Easy calculation shows that

$$
\lim \inf \frac{1}{n} D^{2}\left(\sum_{\imath=1}^{n} f\left(S_{\imath}\right)\right) \geqq \text { const } \cdot D_{B}^{2}(f(S)) \cdot D^{2}\left(N_{\jmath}\right) \cdot \mu(B),
$$

where $D_{B}^{2}$ denotes the variance with respect to the stationary measure restricted to the set $B$. Therefore the positivity of the limiting variance is proved if $D_{B}^{2}(f(S))>0$, and it is easy to see that this is the case for the functions $N_{0}(T), N_{1}(T)$ and $Q(T)$ in Corollaries 1 and 2 .

We want to express our gratitude to Detlef Dürr who was helpful in finding the error in Orey's paper and encouraged us to correct our proof and to Misha Soloveychik for his careful reading of our paper. 